\title{
SELEÇÃO DE INSTÂNCIAS DE GRANDES BASES DE DADOS USANDO ALGORITMOS EVOLUTIVOS MULTIOBJETIVO
}

\author{
João Paulo Santos Sena ${ }^{1}$; Matheus Giovanni Pires ${ }^{2}$; \\ 1. Bolsista PIBIC/CNPq, Graduando em Engenharia de Computação, Universidade Estadual de Feira de Santana, \\ e-mail: joaopaulo761@gmail.com \\ 2. Orientador, Departamento de Exatas, Universidade Estadual de Feira de Santana, e-mail: mgpires@ecomp.uefs.br
}

PALAVRAS-CHAVE: redução de dados; seleção de instâncias; algoritmos genéticos multiobjetivo.

\section{INTRODUÇÃO}

Os Sistemas Baseados em Regras Fuzzy (SBRF) têm sido amplamente usados para a resolução de diversos tipos de problemas, tais como, controle (Leephakpreeda, 2011), modelagem (Pedrycz, 1996), classificação (Ishibuci, 1995). A maneira mais comum para a aquisição do conhecimento de um SBRF é a partir de dados numéricos, os quais representam amostras ou exemplos do problema. As formas mais bem-sucedidas de extração automática de conhecimento a partir de dados para a construção de SFBR são as que combinam metodologias para aprendizado de máquina com conceitos de sistemas fuzzy. Entre elas, destacam-se as Redes Neurais Artificiais e a Computação Evolutiva (Cordón et al, 2001).

Os Algoritmos Genéticos Multiobjetivo (AGMO), vêm demonstrando ser uma poderosa ferramenta para a construção automática (ou projeto automático) de SBRF. No entanto, este processo é fortemente influenciado pela quantidade de instâncias e características presentes nas bases de dados, que afetam o tamanho do espaço de busca e o tempo computacional. Por isso, a redução de dados é de fundamental importância para reduzir o tempo de aprendizado do SBRF e amenizar as dificuldades durante o processo de convergência dos algoritmos evolutivos.

A redução de dados, neste caso, a seleção de instâncias, é um problema multiobjetivo, pois busca-se reduzir a base de dados, e ao mesmo tempo, manter o desempenho do classificador estável ou superior, quando comparado com a base de dados original. Portanto, este trabalho possui o objetivo de investigar a aplicação de Algoritmos Genéticos Multiobjetivo para a seleção de instâncias de grandes bases de dados.

\section{METODOLOGIA}

A seleção de instâncias foi realizada por três AGMO, sendo eles: NSGA-II (Deb et al. 2002), NSGA-III (Deb \& Jain, 2014) e o NSGA-DO (Pimenta \& Camargo, 2015). A implementação destes algoritmos foi realizada utilizando o framework jMetal (Durillo \& Nebro, 2011). O Problema foi modelado considerando uma codificação binária do cromossomo, onde 1 indica a presença de uma instância e 0 sua ausência. Os operadores escolhidos juntamente com suas taxas para cruzamento, mutação e seleção foram Single Point Crossover (0.9), Bit Flip Mutation (0.2) e Binary Tournament, respectivamente. O tamanho da população foi definido como 100 e a quantidade máxima de gerações 50 . No entanto, a execução poderia ser interrompida antes das 50 gerações, caso não houvesse melhora do melhor indivíduo durante cinco gerações seguidas. Os valores dos parâmetros descritos foram definidos de forma empírica. A função de fitness, responsável pela 
avaliação de cada cromossomo, foi definida a partir de dois objetivos, acurácia e taxa de redução, que são obtidos através das equações 1 e 2 respectivamente.

$$
\begin{aligned}
& \text { Acurácia }=\frac{\text { classificações de instâncias corretas }}{\text { quantidade total de instâncias }} \\
& \text { Redução }=\frac{\text { quantidade total }- \text { quantidade selecionada }}{\text { quantidade total }}
\end{aligned}
$$

O classificador utilizado para o cálculo da acurácia durante o treinamento foi o $k$ Nearest Neighbor (KNN) disponível no WEKA ${ }^{1}$, com o valor de $k$ igual a 5.

Após a aplicação do AGMO para a seleção de instancias foram utilizados os classificadores $\mathrm{KNN}$ e o C4.5, que se baseia em uma arvore de decisões, como classificadores de testes, ambos disponíveis no WEKA ${ }^{1}$.

Como o resultado do AGMO é um conjunto de soluções, escolheu-se a solução média entre os 2 objetivos, não priorizando a acurácia nem a redução.

\section{RESULTADOS E/OU DISCUSSÃO}

Para a realização dos experimentos foram utilizadas 37 bases de dados extraídas do repositório aberto de dados do $\mathrm{KEEL}^{2}$. No entanto neste resumo serão apresentados os resultados apenas de 9 bases, as quais foram selecionadas aleatoriamente, devido ao espaço limitado deste resumo. Elas foram divididas em conjuntos de treinamento e teste, utilizando a abordagem 10-fold cross validation (Kohavi, 1995). Além disso, cada fold foi executado três vezes, logo, para cada base de dados foram efetuadas 30 execuções. Portanto, os resultados expressam a média destas 30 execuções. Baseado no trabalho de Fazzolari et al. (2013), as bases também foram reduzidas utilizando o algoritmo PBIL e os resultados foram comparados com os AGMO.

Para comparar os resultados obtidos foi utilizado o teste de Friedman (Friedman, 1940) para verificar se existem diferenças significantes entre os resultados. Uma vez constatada uma diferença significante, os resultados são comparados em pares, utilizando o teste Wilcoxon Signed-Ranks (Wilcoxon, 1945).

Os resultados obtidos estão descritos nas Tabelas 1, 2 e 3, as quais, mostram a média e desvio padrão das medidas de tempo, acurácia e taxa de redução, respectivamente. O tempo de classificação foi calculado em segundos.

De acordo com os resultados da Tabela 1, é possível constatar uma redução no tempo de classificação, considerando as bases reduzidas pelos AGMO, quando comparados com a base de tamanho original. Por outro lado, ao se comparar os tempos de classificação com a base reduzida pelo algoritmo PBIL, este proporcionou uma classificação mais rápida em relação aos AGMO.

Os dados da Tabela 1 estão relacionados com os da Tabela 2, pois a redução no tempo de classificação está diretamente relacionada com a redução das bases. Ao analisar

\footnotetext{
${ }^{1}$ WEKA: Waikato Environment for Knowledge Analysis. https://www.cs. waikato.ac.nz/ml/weka

${ }^{2} \mathrm{http}: / /$ sci2s.ugr.es/keel/ datasets.php
} 
os resultados da Tabela 2, verifica-se que a redução obtida pelos AGMO ficou em torno de $50 \%$ da base original, enquanto que o PBIL ficou em torno de $89 \%$.

Tabela 1. Tempo de classificação

\begin{tabular}{|c|c|c|c|c|c|c|c|c|c|c|}
\hline & \multicolumn{2}{|c|}{ NSGAII } & \multicolumn{2}{|c|}{ NSGAIII } & \multicolumn{2}{|c|}{ NSGA-DO } & \multicolumn{2}{|c|}{ PBIL } & \multicolumn{2}{|c|}{ Original } \\
\hline & Média & Desvio & Média & Desvio & Média & Desvio & Média & Desvio & Média & Desvio \\
\hline coil2000 & 347.9 & 32.0 & 348.5 & 31.5 & 369.2 & 58.0 & 29.6 & 8.7 & 1218.9 & 137.2 \\
\hline magic & 176.6 & 26.4 & 171.9 & 26.2 & 176.6 & 21.1 & 36.1 & 7.1 & 507.9 & 109.7 \\
\hline marketing & 102.0 & 8.8 & 102.6 & 9.7 & 106.7 & 11.4 & 12.4 & 6.2 & 307.8 & 31.9 \\
\hline optdigits & 115.1 & 7.6 & 119.8 & 11.6 & 117.7 & 11.2 & 15.6 & 6.9 & 306.2 & 7.7 \\
\hline penbased & 91.6 & 12.6 & 100.5 & 18.7 & 95.8 & 8.8 & 15.7 & 0.5 & 217.1 & 16.4 \\
\hline ring & 180.7 & 16.4 & 174.5 & 11.6 & 178.0 & 14.3 & 23.2 & 7.8 & 492.2 & 39.2 \\
\hline Satimage & 110.5 & 8.0 & 114.1 & 13.5 & 108.8 & 7.5 & 15.8 & 0.4 & 273.5 & 7.9 \\
\hline Texture & 105.2 & 8.0 & 106.3 & 9.4 & 105.2 & 8.1 & 14.2 & 4.7 & 242.1 & 7.9 \\
\hline Twonorm & 84.9 & 7.7 & 86.4 & 8.8 & 86.5 & 7.8 & 10.8 & 7.1 & 229.8 & 12.1 \\
\hline
\end{tabular}

Tabela 2. Taxas de redução alcançadas pelos algoritmos nas bases grandes.

NSGAIII

NSGA-DO

PBIL

\begin{tabular}{lcccccccc} 
& Média & Desvio & Média & Desvio & Média & Desvio & Média & Desvio \\
\cline { 2 - 8 } coil2000 & $51.7 \%$ & $0.4 \%$ & $51.8 \%$ & $0.4 \%$ & $51.6 \%$ & $0.4 \%$ & $88.1 \%$ & $0.3 \%$ \\
magic & $50.8 \%$ & $0.7 \%$ & $50.8 \%$ & $0.6 \%$ & $50.7 \%$ & $0.6 \%$ & $85.5 \%$ & $0.2 \%$ \\
marketing & $51.2 \%$ & $0.7 \%$ & $51.1 \%$ & $0.8 \%$ & $50.9 \%$ & $0.5 \%$ & $87.5 \%$ & $0.2 \%$ \\
optdigits & $51.8 \%$ & $0.4 \%$ & $51.9 \%$ & $0.5 \%$ & $52.0 \%$ & $0.6 \%$ & $91.2 \%$ & $0.2 \%$ \\
penbased & $51.4 \%$ & $0.4 \%$ & $51.5 \%$ & $0.4 \%$ & $51.5 \%$ & $0.3 \%$ & $88.9 \%$ & $0.2 \%$ \\
ring & $51.2 \%$ & $1.2 \%$ & $51.7 \%$ & $1.4 \%$ & $51.5 \%$ & $1.2 \%$ & $86.7 \%$ & $0.2 \%$ \\
satimage & $51.3 \%$ & $0.8 \%$ & $51.1 \%$ & $1.1 \%$ & $51.8 \%$ & $0.9 \%$ & $89.5 \%$ & $0.1 \%$ \\
texture & $51.6 \%$ & $0.6 \%$ & $51.7 \%$ & $0.8 \%$ & $51.8 \%$ & $0.7 \%$ & $90.7 \%$ & $0.3 \%$ \\
twonorm & $51.6 \%$ & $0.6 \%$ & $51.6 \%$ & $0.6 \%$ & $51.7 \%$ & $0.6 \%$ & $89.6 \%$ & $0.2 \%$ \\
\hline
\end{tabular}

Tabela 3. Acurácia das bases de dados reduzidas comparas com a base original

\begin{tabular}{lcccccccccc} 
& \multicolumn{2}{c}{ NSGAll } & \multicolumn{2}{c}{ NSGAllI } & \multicolumn{2}{c}{ NSGA-DO } & \multicolumn{2}{c}{ PBIL } & \multicolumn{2}{c}{ Original } \\
& Média & Desvio & Média & Desvio & Média & Desvio & Média & Desvio & Média & Desvio \\
\cline { 2 - 10 } coil2000 & 0.94 & 0.00 & 0.94 & 0.00 & 0.94 & 0.00 & 0.94 & 0.00 & 0.94 & 0.00 \\
magic & 0.85 & 0.01 & 0.85 & 0.01 & 0.85 & 0.01 & 0.84 & 0.01 & 0.85 & 0.01 \\
marketing & 0.30 & 0.02 & 0.30 & 0.02 & 0.30 & 0.02 & 0.36 & 0.02 & 0.31 & 0.02 \\
optdigits & 0.88 & 0.01 & 0.88 & 0.01 & 0.88 & 0.01 & 0.80 & 0.01 & 0.91 & 0.01 \\
penbased & 0.95 & 0.01 & 0.95 & 0.01 & 0.95 & 0.01 & 0.91 & 0.01 & 0.96 & 0.01 \\
ring & 0.89 & 0.01 & 0.89 & 0.01 & 0.89 & 0.01 & 0.85 & 0.02 & 0.91 & 0.01 \\
satimage & 0.85 & 0.02 & 0.85 & 0.01 & 0.85 & 0.01 & 0.84 & 0.01 & 0.86 & 0.01 \\
texture & 0.91 & 0.01 & 0.91 & 0.01 & 0.90 & 0.01 & 0.85 & 0.02 & 0.93 & 0.01 \\
twonorm & 0.84 & 0.01 & 0.84 & 0.01 & 0.84 & 0.01 & 0.82 & 0.01 & 0.85 & 0.01 \\
\hline
\end{tabular}

Por fim é necessário analisar se a redução das bases impactou no desempenho do classificador. Na Tabela 3 é possível verificar que a redução dos dados não impactou na classificação, pois os resultados são próximos ou iguais quando comparados com a base de dados de tamanho original. De acordo com o método estatístico de Friedman, não 
houveram diferenças estatísticas na acurácia, quando comparados as bases de dados reduzidas pelos AGMO e PBIL com a original.

\section{CONSIDERAÇÕES FINAIS}

Este trabalho teve como objetivo analisar o impacto da classificação de bases de dados reduzidas por AGMO. Os resultados mostram que todas as bases de dados foram reduzidas em aproximadamente $50 \%$ e apesar da redução o desempenho dos classificadores não foi prejudicado de acordo com o método de Friedman. Deste modo, a seleção de instâncias, utilizando AGMO torna-se uma alternativa para obter um subconjunto reduzido de dados com o mesmo desempenho do conjunto original.

Como trabalhos futuros, as bases de dados reduzidas neste trabalho serão utilizadas para a construção de Sistemas Baseados em Regras Fuzzy.

\section{REFERÊNCIAS}

FRIEDMAN, Milton (1940). A comparison of alternative tests of significance for the problem of m rankings. The Annals of Mathematical Statistics, v. 11, n. 1, p. 86-92.

WILCOXON, Frank (1945) Individual comparisons by ranking methods. Biometrics bulletin, v. 1, n. 6, p. 80-83.

ISHIBUCHI, H., NOZAKI, K., YAKAMOTO, N. e TANAKA, H. (1995). Selecting Fuzzy If-Then Rules for Classification Problems using Genetic Algorithms. IEEE Transactions on Fuzzy Systems, vol.3, n.3, pp.260-270.

KOHAVI, Ron et al. (1995). A study of cross-validation and bootstrap for accuracy estimation and model selection. International Joint Conference on Artificial intelligence, p. 1137-1145.

PEDRYCZ, W. (1996). Fuzzy modelling: Paradigms and practice, Kluwer Academic Publishers.

CORDÓN, O., HERRERA, F. e VILLAR, P. (2001). Generating the Knowledge Base of a Fuzzy Rule-Based System by the Genetic Learning of the Data Base. IEEE Transactions on Fuzzy Systems, vol.9, n.4, pp.667-674.

DEB, K., PRATAP, A., AGARWAL, S., MEYARIVAN, T. (2002). A Fast and Elitist Multiobjective Genetic Algorithm: NSGA-II. IEEE Transactions on Evolutionary Computation, vol.6, n.2, pp.182-197.

LEEPHAKPREEDA, T. (2011). Fuzzy logic based PWM control and neural controlledvariable estimation of pneumatic artificial muscle actuators. Expert Systems with Applications, vol.38, n.6, pp.7837-7850.

JUAN A. J. N., DURILLO, J. (2011). jMetal: A Java framework for multiobjective optimization, Advances in Engineering Software, p. 760-771.

FAZZOLARI, M., GIGLIO, B., ALCALÁ, R., MARCELlONI, F. HERRERA, F. (2013). A study on the application of instance selection techniques in genetic fuzzy rulebased classification systems: Accuracy-complexity trade-off. Knowledge-Based Systems, vol.54, pp.32-41.

DEB, K., JAIN, H. (2014) An Evolutionary Many-Objective Optimization Algorithm Using Reference-Point-Based Nondominated Sorting Approach, Part I: Solving Problems with Box Constraints. IEEE Transactions on Evolutionary Computation, vol.18, no.4.

PIMENTA, A. H. M. e CAMARGO, H. A. (2015). NSGA-DO: Non-Dominated Sorting Genetic Algorithm Distance Oriented. IEEE International Conference on Fuzzy Systems, pp.1-8. 